\title{
SOME INTEGRAL INEQUALITIES WITH BOUNDS FOR MOMENTS OF DISTRIBUTION II
}

\author{
SANJA VAROŠANEC ${ }^{1}$ and JOSIP PEČARIĆ ${ }^{2}$
}

(Received 24 January 1997; revised 20 January 1999)

\begin{abstract}
Several generalizations are given of the Gauss-Winckler inequality for the moments of a probability distribution.
\end{abstract}

\section{Introduction}

Bounds on the moments of probability distributions are important in the area of stochastic analysis generally and also in operations research. In this article we extend several known results.

To fix our notation, let $Q:[0, \alpha] \rightarrow[0,1](\alpha \in(0, \infty])$ be a nondecreasing function with $Q(0)=0$ and $Q(\alpha)=1$, that is, $Q$ is a probability distribution function with support $[0, \alpha]$. The $r$-th moment $\nu_{r}$ of $Q$ is defined by

$$
v_{r}=\int_{0}^{\alpha} x^{r} d Q(x)
$$

This is naturally defined for $r \geq 0$, though we shall find that a number of results actually hold for $r>-1$. In the remainder of this paper we assume that $Q$ has the above-mentioned properties.

In recent years a rich variety of relations has been uncovered between the moments of distributions. The roots of this go back over a century. Thus, under the additional constraint of a nonincreasing density function, the Gauss-Winckler inequality says that

$$
\left((r+1) v_{r}\right)^{1 / r} \leq\left((s+1) v_{s}\right)^{1 / s} \text { for } r<s .
$$

\footnotetext{
${ }^{1}$ Department of Mathematics, University of Zagreb, Bijenička cesta 30, 10000 Zagreb, Croatia.

${ }^{2}$ Faculty of Textile Technology, University of Zagreb, Pierottijeva 6, 10000 Zagreb, Croatia.

(C) Australian Mathematical Society 2000, Serial-fee code 0334-2700/00
} 
When $r=1$ and $s=2$, this gives

$$
v_{2} \geq(4 / 3) v_{1}^{2}
$$

which is a surprising and useful strengthening of the familiar $v_{2} \geq v_{1}^{2}$.

Slightly more involved is the following theorem of the authors $[6,9]$.

THEOREM A. $\quad 1^{\circ}$ Let $\alpha=1$ and suppose $Q$ has a derivative of order $(n+1)$ such that $(-1)^{n+1} Q^{(n+1)}$ is nonnegative.

(a) For $n \in\{1,2\}$ the function $f_{n}$ defined by

$$
f_{n}(r)=\ln \left[\left(\begin{array}{c}
r+n \\
n
\end{array}\right) v_{r}\right]
$$

is concave on $(-1, \infty)$.

(b) If $n \geq 3$ and $(-1)^{n-k-1} Q^{(n-k)}(1) \geq 0$ for $k=2, \ldots, n-1$, then $f_{n}$ is concave also.

$2^{\circ}$ If $\alpha \in \mathbf{R}$ and if $Q^{\prime}$ is nondecreasing, then $f_{1}$ is concave.

For $n=1$, part (a) says that if a distribution on a bounded interval (which we may take as $[0,1])$ has a monotone nondecreasing density function, then $(r+1) v_{r}$ is logconcave on $(-1, \infty)$. The same conclusion holds (for $n=2$ ) if the distribution has a concave density function.

Similarly we derived the following result.

THEOREM B. (a) If $\alpha=\infty$ and $(-1)^{n-1} Q^{(n)}$ is positive, continuous and nonincreasing for $n=1,2 \ldots, N$, then $f_{n}(n=1,2, \ldots, N)$ is convex.

(b) If $\alpha \in \mathbf{R}$ and $Q^{\prime}$ is nonincreasing, then $f_{1}$ is convex.

As a consequence of the concavity of $f_{n}$ in Theorem $\mathrm{A}$, we have for $r \geq 0$ and $r_{1}, \ldots, r_{m} \geq 0$ that

$$
\left[\left(\begin{array}{c}
r+n \\
n
\end{array}\right) v_{r}\right]^{m-1}\left(\begin{array}{c}
r_{1}+\cdots+r_{m}+r+n \\
n
\end{array}\right) v_{r_{1}+\cdots+r_{m}+r} \leq \prod_{i=1}^{m}\left(\begin{array}{c}
r_{i}+r+n \\
n
\end{array}\right) v_{r_{i}+r}
$$

When $f_{n}$ is convex, the reverse of (1) holds. For $n=1$, the reverse of (1) was proved by Pečarić [5] using Chebyshev's inequality. It is an extension of Stolarsky's inequality ([7], see also [6, 4] and Section 4).

These results are suggestive of much more. We explore some of the possibilities in this paper.

In Section 2 we give a weighted version of (1) and connect it with the Petrovic inequality. 
In Section 3 we use a similar idea to derive generalizations of a result related to the Gauss-Winckler inequality. These involve integrals $\int_{0}^{1} f^{r}(x) d x$, where $f(Q(x)) / x$ is nondecreasing. In Section 4 we take up the common case of an exponential distribution, standardised to unit mean, to derive new inequalities for the gamma function. A simple choice of distribution on a finite interval similarly gives results for the beta function. These are, of course, intimately associated with the gamma and beta distributions. Finally, in Section 5, we derive an improvement of Stolarsky's inequality.

\section{Weighted inequality}

THEOREM 1. Let $f_{n}$ be a concave function (that is, the conclusions of Theorem A hold $)$. If $r, p_{i}, r_{i}(i=1, \ldots, m)$ are real numbers such that $\sum_{i=1}^{m} p_{i} r_{i}+r, r_{j}+r$ $(j=1, \ldots, m)$ and $r$ are greater than $-1, p_{i} \geq 0(i=1, \ldots m)$ and

$$
r_{j} \cdot\left(\sum_{i=1}^{m} p_{i} r_{i}-r_{j}\right) \geq 0, j=1, \ldots, m
$$

then

$$
\left[\left(\begin{array}{c}
r+n \\
n
\end{array}\right) v_{r}\right]^{P_{m}-1}\left(\begin{array}{c}
\sum_{i=1}^{m} p_{i} r_{i}+r+n \\
n
\end{array}\right) v_{\sum_{i=1}^{m} \rho_{i} r_{i}+r} \leq \prod_{j=1}^{m}\left[\left(\begin{array}{c}
r_{j}+r+n \\
n
\end{array}\right) v_{r_{j}+r}\right]^{p_{j}},
$$

where $P_{m}=\sum_{i=1}^{m} p_{i}$.

If $r_{j} \cdot\left(\sum_{i=1}^{m} p_{i} r_{i}-r_{j}\right) \leq 0(j=1, \ldots, m)$, then the inequality is reversed.

Proof. Suppose (2) holds. Since $f_{n}$ is concave, for any $p, q, s>-1, p \geq q$, $p \neq s, q \neq s$, we have

$$
\frac{f_{n}(p)-f_{n}(s)}{p-s} \leq \frac{f_{n}(q)-f_{n}(s)}{q-s}
$$

(see $[3$, p. 2]).

Thus for $p, q, s>-1, p \geq q, p \neq s, q \neq s$, we get

$$
\left(\frac{\left(\begin{array}{c}
p+n \\
n
\end{array}\right) \nu_{p}}{\left(\begin{array}{c}
s+n \\
n
\end{array}\right) \nu_{s}}\right)^{1 /(p-s)} \leq\left(\frac{\left(\begin{array}{c}
q+n \\
n
\end{array}\right) \nu_{q}}{\left(\begin{array}{c}
s+n \\
n
\end{array}\right) \nu_{s}}\right)^{1 /(q-s)}
$$

If $r_{j}>0$ then $\sum_{i=1}^{m} p_{i} r_{i}>r_{j}$ and setting $p=\sum_{i=1}^{m} p_{i} r_{i}+r, q=r_{j}+r$ and $s=r$ in 
(4) we obtain

$$
\begin{aligned}
& \left(\frac{\left(\begin{array}{c}
\sum_{i=1}^{m} p_{i} r_{i}+r+n \\
n
\end{array}\right) \nu_{\sum_{i=1}^{m} p_{i} r_{i}+r}}{\left(\begin{array}{c}
r n \\
n
\end{array}\right) \nu_{r}}\right)^{1 / \sum_{i=1}^{m} p_{i} r_{i}} \leq\left(\frac{\left(\begin{array}{c}
r_{j}+r+n \\
n
\end{array}\right) \nu_{r_{j}+r}}{\left(\begin{array}{c}
r+n \\
n
\end{array}\right) \nu_{r}}\right)^{1 / r_{j}} \\
& \left(\frac{\left(\begin{array}{c}
\sum_{i=1}^{m} p_{i} r_{i}+r+n \\
n
\end{array}\right) \nu_{\sum_{i=1}^{m} p_{i} r_{i}+r}}{\left(\begin{array}{c}
r+n \\
n
\end{array}\right) \nu_{r}}\right)^{p_{j} r_{j} / \sum_{i=1}^{m} p_{i} r_{i}} \leq\left(\frac{\left(\begin{array}{c}
r_{j}+r+n \\
n
\end{array}\right) \nu_{r_{j}+r}}{\left(\begin{array}{c}
r+n \\
n
\end{array}\right) \nu_{r}}\right)^{p_{j}} \text {. }
\end{aligned}
$$

If $r_{j}<0$ then $\sum_{i=1}^{m} p_{i} r_{i}<r_{j}$ and setting $p=r_{j}+r, q=\sum_{i=1}^{m} p_{i} r_{i}+r$ and $s=r$ in (4) we obtain (5).

By multiplication of these inequalities for $j=1, \ldots, m$ we get (3). The other cases can be proved analogously.

REMARK 1. If $p_{i}=1, r_{i} \geq 0(i=1, \ldots, m)$ then (3) reduces to (1).

REMARK 2. The Petrović inequality (see $[3$, p. 11]) states the following.

Suppose $f$ is convex on $I=[a, b](0 \in I)$ and $r_{i}, p_{i}(i=1, \ldots, m)$ are real numbers such that $r_{i} \sum_{i=1}^{m} p_{i} r_{i} \in I\left(p_{i} \geq 0\right)$ for $i=1, \ldots, m$. If (2) holds, then

$$
f\left(\sum_{i=1}^{m} p_{i} r_{i}\right) \geq \sum_{i=1}^{m} p_{i} f\left(r_{i}\right)+\left(1-P_{m}\right) f(0) .
$$

If $f$ is concave, the inequality is reversed.

In our case $f_{n}$ is concave and $f_{n}(0)=\ln \nu_{0}=0$, so the inequality becomes

$$
\sum_{i=1}^{m}\left(\begin{array}{c}
p_{i} r_{i}+n \\
n
\end{array}\right) v_{\sum_{i=1}^{m} p_{i} r_{i}+n} \geq \prod_{i=1}^{m}\left(\left(\begin{array}{c}
r_{i}+n \\
n
\end{array}\right) v_{r_{i}}\right)^{p_{i}}
$$

Also putting $r=0$ in (3) gives (6).

REMARK 3. When $f_{n}$ is convex, that is, when the conclusion of Theorem B holds, then the inequality is reversed.

\section{Some further results}

The following theorem was proved in [2].

THEOREM 2. Let $f:[0,1] \rightarrow \mathbf{R}$ be nondecreasing and positive. If $f(Q(x)) / x$ is nondecreasing, then for $m \leq r$

$$
\frac{v_{r}^{1 / r}}{v_{m}^{1 / m}} \leq \frac{\left(\int_{0}^{1} f^{r}(x) d x\right)^{1 / r}}{\left(\int_{0}^{1} f^{m}(x) d x\right)^{1 / m}}
$$


If $f(Q(x)) / x$ is nonincreasing, then the inequality is reversed.

Using the idea in the proof of Theorem 1, we obtain the following.

THEOREM 3. Let $f:[0,1] \rightarrow \mathbf{R}$ be nondecreasing and positive. Let $p_{i}(i=$ $1, \ldots, m)$ be positive real numbers and $r_{i}(i=1, \ldots, m)$ real numbers such that $r_{i}$ $(i=1, \ldots, m)$ and $\sum_{i=1}^{m} p_{i} r_{i}$ are greater than -1 . If $f(Q(x)) / x$ is nondecreasing and if $r_{j}\left(\sum_{i=1}^{m} p_{i} r_{i}-r_{j}\right) \geq 0(j=1, \ldots, m)$, then

$$
\frac{\nu_{\sum_{i=1}^{m} p_{i} r_{r}}}{\prod_{i=1}^{m} v_{r_{j}}^{j_{j}}} \leq \frac{\int_{0}^{1} f^{\sum_{i=1}^{m} p_{i} r_{i}}(x) d x}{\prod_{i=1}^{m}\left(\int_{0}^{l} f^{r_{j}}(x) d x\right)^{p_{j}}} .
$$

If $r_{j}\left(\sum_{i=1}^{m} p_{i} r_{i}-r_{j}\right) \leq 0(j=1, \ldots, m)$, the inequality (7) is reversed.

If $f(Q(x)) / x$ is nonincreasing, the above results are reversed.

Let us mention some special cases when (2) holds.

$1^{\circ} \quad$ For $f \equiv 1$ we have $\nu_{p_{1} r_{1}+\cdots+p_{m} r_{m}} \geq v_{r_{1}}^{p_{1}} \cdots \nu_{r_{m}}^{p_{m}}$.

$2^{\circ}$ For $f(x)=x$ and $Q(x) / x$ nondecreasing, we have

$$
\left(\sum_{i=1}^{m} p_{i} r_{i}+1\right) v_{\sum_{i=1}^{m} p_{i} r_{i}} \leq \prod_{j=1}^{m}\left(r_{j}+1\right)^{p_{j}} \nu_{r_{j}}^{p_{j}},
$$

while if $Q(x) / x$ is nonincreasing, the opposite inequality holds.

$3^{\circ}$ For $f(x)=x^{1 / k}(k>0)$ and $Q(x) / x^{k}$ nondecreasing, we have

$$
\frac{p_{1} r_{1}+\cdots+p_{m} r_{m}+k}{k} v_{p_{1} r_{1}+\cdots+p_{m} r_{m}} \leq \prod_{j=1}^{m}\left(\frac{r_{j}+k}{k}\right)^{p_{j}} \nu_{r_{j}}^{p_{j}} .
$$

When $Q(x) / x^{k}$ is nonincreasing the opposite inequality holds. The case $p_{1}=p_{2}=$ $\cdots=p_{m}=1$ can be found in [6].

\section{The gamma and beta functions}

If $\alpha=\infty$ and $Q(x)=1-e^{-x}$, then $Q$ satisfies the assumptions of Theorem B(a) for any $N \in \mathbf{N}$, so $f_{n}$ is convex for any $n \in \mathbf{N}$. In this case $\nu_{r}=\Gamma(r+1)$ and we derive the following inequalities for the gamma function.

COROLlaRY 1. (a) Suppose $p_{i}(i=1, \ldots, m)$ are positive real numbers and $r, r_{i}(i=1, \ldots, m)$ are real numbers such that $r>-1, \sum_{i=1}^{m} p_{i} r_{i}+r>-1$ and 
$r_{j}+r>-1$ for $j=1, \ldots$, m. If (2) holds, then

$$
\begin{gathered}
\left(\left(\begin{array}{c}
r+n \\
n
\end{array}\right) \Gamma(r+1)\right)^{P_{m}-1}\left(\begin{array}{c}
\sum_{i=1}^{m} p_{i} r_{i}+r+n \\
n
\end{array}\right) \Gamma\left(\sum_{i=1}^{m} p_{i} r_{i}+r+1\right) \\
\geq \prod_{j=1}^{m}\left(\begin{array}{c}
r_{j}+r+n \\
n
\end{array}\right)^{p_{j}} \Gamma^{p_{j}}\left(r_{j}+r+1\right) .
\end{gathered}
$$

(b) If $p_{i}(i=1, \ldots, m)$ are positive real numbers and $r_{i}(i=1, \ldots, m)$ real numbers such that $r_{i}(i=1, \ldots, m)$ and $\sum_{i=1}^{m} p_{i} r_{i}$ are greater than -1 , then

$$
\Gamma\left(\sum_{i=1}^{m} p_{i} r_{i}+1\right) \geq \prod_{j=1}^{m} \Gamma^{p_{j}}\left(r_{j}+1\right)
$$

and

$$
\frac{\sum_{i=1}^{m} p_{i} r_{i}+k}{k} \Gamma\left(\sum_{i=1}^{m} p_{i} r_{i}+1\right) \geq \prod_{j=1}^{m}\left(\frac{r_{j}+k}{k}\right)^{p_{j}} \Gamma^{p_{j}}\left(r_{j}+1\right)
$$

for $k \geq 1$.

If inequality (2) is reversed, then so are (8)-(10).

If $\alpha=1$ and $Q(x)=1-(1-x)^{a+1}(a>0)$, then $Q$ satisfies the assumptions of Theorem $\mathrm{B}(\mathrm{b})$ and we derive inequalities for the beta function.

COROllaRY 2. Suppose $p_{i}(i=1, \ldots, m)$ are positive real numbers and $r, r_{i}$ $(i=1, \ldots, m)$ are real numbers such that $r>-1, \sum_{i=1}^{m} p_{i} r_{i}+r>-1$ and $r_{j}+r>-1$ for $j=1, \ldots, m$. If (2) holds, then

$$
\begin{aligned}
& ((r+1) B(r+1, a+1))^{P_{m}-1}\left(\sum_{i=1}^{m} p_{i} r_{i}+r+1\right) B\left(\sum_{i=1}^{m} p_{i} r_{i}+r+1, a+1\right) \\
& \geq \prod_{j=1}^{m}\left(r_{j}+r+1\right)^{p_{j}} B^{p_{j}}\left(r_{j}+r+1, a+1\right),
\end{aligned}
$$

where $B(x+1, y+1)=\int_{0}^{1} t^{x}(1-t)^{y} d t$

(b) If $p_{i}(i=1, \ldots, m)$ are positive real numbers and $r_{i}(i=1, \ldots, m)$ real numbers such that $r_{i}(i=1, \ldots, m)$ and $\sum_{i=1}^{m} p_{i} r_{i}$ are greater than -1 , then

$$
B\left(\sum_{i=1}^{m} p_{i} r_{i}+1, a+1\right) \geq(a+1)^{P_{m}-1} \prod_{j=1}^{m} B^{p_{j}}\left(r_{j}+1, a+1\right)
$$


and

$$
\begin{aligned}
& {\left[\sum_{i=1}^{m} p_{i} r_{i}+1\right] B\left(\sum_{i=1}^{m} p_{i} r_{i}+1, a+1\right)} \\
& \quad \geq(a+1)^{P_{m}-1} \prod_{j=1}^{m}\left(r_{j}+1\right)^{p_{j}} B^{p_{j}}\left(r_{j}+1, a+1\right) .
\end{aligned}
$$

Reversal of the direction of inequality (2) reverses those of the above conclusions.

\section{Integral inequalities involving derivatives}

Stolarsky [7] has established the following theorem.

THEOREM C. If $f:[0,1] \rightarrow[0,1]$ is nonnegative and nonincreasing, then for positive $a$ and $b$

$$
(a+b) \int_{0}^{1} x^{a+b-1} f(x) d x \geq a b \int_{0}^{1} x^{a-1} f(x) d x \int_{0}^{1} x^{b-1} f(x) d x
$$

In probabilistic terms, this gives the following. Suppose $\int_{0}^{1} f(x) d x=1$, that is, $f$ is a probability density on $[0,1]$. If this is nondecreasing, then

$$
(a+b) v_{a+b-1} \geq a b v_{a-1} v_{b-1}
$$

In the first section we mentioned that (1) is an extension of Stolarsky's result (11). In this section we give another improvement of (11).

THEOREM 4. Let $f:[a, b] \rightarrow \mathbf{R}$ be of bounded variation and $g, h:[a, b] \rightarrow \mathbf{R}$ nonnegative and nondecreasing with continuous first derivative and $g(a)=h(a)=0$. If $0 \leq f(b) \leq f(x) \leq f(a)$ for all $x \in[a, b]$, then

$$
f(a) \int_{a}^{b}(g(t) h(t))^{\prime} f(t) d t \geq \int_{a}^{b} g^{\prime}(t) f(t) d t \int_{a}^{b} h^{\prime}(t) f(t) d t
$$

If $0 \leq f(a) \leq f(x) \leq f(b)$, the inequality is reversed.

PROOF. If $0 \leq f(b) \leq f(x) \leq f(a)$, then we may without loss of generality assume that $f(b)<f(a)$, because if $f(b)=f(a)$, then $f \equiv C$ and (12) holds. The following inequality of Chebyshev type, established in [1], will be used. 
If $f_{1}, f_{2}:[a, b] \rightarrow \mathbf{R}$ are nondecreasing and continuous and $f:[a, b] \rightarrow \mathbf{R}$ of bounded variation such that $f(a) \leq f(x) \leq f(b)$ for all $x \in[a, b]$, then

$$
\int_{a}^{b} d f(x) \int_{a}^{b} f_{1}(x) f_{2}(x) d f(x) \geq \int_{a}^{b} f_{1}(x) d f(x) \int_{a}^{b} f_{2}(x) d f(x) .
$$

Now integration by parts and (13) give

$$
\begin{aligned}
& \int_{a}^{b}(g(t) h(t))^{\prime} f(t) d t \\
& \quad=f(b) g(b) h(b)+\int_{a}^{b} g(t) h(t) d(-f)(t) \\
& \quad \geq f(b) g(b) h(b)+[f(a)-f(b)]^{-1} \int_{a}^{b} g(t) d(-f)(t) \int_{a}^{b} h(t) d(-f)(t)
\end{aligned}
$$

From the identity

$$
\left(a_{1}-a_{2}\right)\left(b_{1}-b_{2}\right) p_{1} p_{2}=\left(p_{1}+p_{2}\right)\left(a_{1} b_{1} p_{1}+a_{2} b_{2} p_{2}\right)-\left(a_{1} p_{1}+a_{2} p_{2}\right)\left(b_{1} p_{1}+b_{1} p_{2}\right)
$$

we have

$$
\left(p_{1}+p_{2}\right)\left(p_{1} a_{1} b_{1}+p_{2} a_{2} b_{2}\right) \geq\left(p_{1} a_{1}+p_{2} a_{2}\right)\left(p_{1} b_{1}+p_{1} b_{2}\right)
$$

for $\left(a_{1} \geq a_{2}, b_{1} \geq b_{2}\right)$ or $\left(a_{1} \leq a_{2}, b_{1} \leq b_{2}\right)$ and $p_{1}, p_{2} \geq 0$. This is the discrete Chebyshev inequality.

We now set

$$
\begin{gathered}
p_{1}=f(b), \quad a_{1}=g(b), \quad b_{1}=h(b), \quad p_{2}=f(a)-f(b), \\
a_{2}=\frac{1}{f(a)-f(b)} \int_{a}^{b} g(t) d(-f)(t), \quad b_{2}=\frac{1}{f(a)-f(b)} \int_{a}^{b} h(t) d(-f)(t)
\end{gathered}
$$

in (15). To use (16), we need only check that $a_{1} \geq a_{2}$ and $b_{1} \geq b_{2}$. We have

$$
\begin{aligned}
\left(a_{1}-a_{2}\right)(f(a)-f(b)) & =g(b)(f(a)-f(b))-\left.g(t)(-f)(t)\right|_{a} ^{b}-\int_{a}^{b} f(t) g^{\prime}(t) d t \\
& =f(a) g(b)-\int_{a}^{b} f(t) g^{\prime}(t) d t=\int_{a}^{b}(f(a)-f(t)) g^{\prime}(t) d t .
\end{aligned}
$$

By assumption the last expression is nonnegative, so $a_{1} \geq a_{2}$. The other statement is proved similarly. 
We combine (14) and (16) to obtain

$$
\begin{aligned}
\int_{a}^{b}(g(t) h(t))^{\prime} f(t) d t & \\
\quad & \geq \frac{1}{f(a)}\left(f(b) g(b)-\int_{a}^{b} g(t) d f(t)\right)\left(f(b) h(b)-\int_{a}^{b} h(t) d f(t)\right) \\
\quad & =\frac{1}{f(a)} \int_{a}^{b} g^{\prime}(t) f(t) d t \int_{a}^{b} h^{\prime}(t) f(t) d t
\end{aligned}
$$

which establishes the first part of the proof.

If $0 \leq f(a) \leq f(x) \leq f(b)$ for all $x \in[a, b]$, a similar proof applies, the only difference being that in place of (16) we use

$$
\left(p_{1}+p_{2}\right)\left(p_{1} a_{1} b_{1}+p_{2} a_{2} b_{2}\right) \leq\left(p_{1} a_{1}+p_{2} a_{2}\right)\left(p_{1} b_{1}+p_{1} b_{2}\right)
$$

for $p_{1} \geq 0, p_{2} \leq 0$ and $a_{1} \geq a_{2}, b_{1} \geq b_{2}$. This is a consequence of (15).

REMARK 4. If $0 \leq f(b) \leq f(x) \leq f(a)$ for all $x \in[a, b]$ and $f, g, h$ satisfy the assumptions of Theorem 4 , then the condition $g(a)=h(a)=0$ can be omitted. For define functions $\bar{h}$ and $\bar{g}$ by

$$
\bar{h}(x)=h(x)-h(a) \quad \text { and } \quad \bar{g}(x)=g(x)-g(a) .
$$

Then $\bar{h}(a)=\bar{g}(a)=0$ and $\bar{h}, \bar{g}$ satisfy the assumptions of Theorem 4 , so

$$
\begin{aligned}
f(a) & \int_{a}^{b}(g(t) h(t))^{\prime} f(t) d t \\
& =f(a) \int_{a}^{b}(\bar{g}(t) \bar{h}(t))^{\prime} f(t) d t+\int_{a}^{b}\left(h(a) g^{\prime}(t)+g(a) h^{\prime}(t)\right) f(t) d t \\
& \geq f(a) \int_{a}^{b}(\bar{g}(t) \bar{h}(t))^{\prime} f(t) d t \geq f(a) \int_{a}^{b} g^{\prime}(t) f(t) d t \int_{a}^{b} h^{\prime}(t) f(t) d t
\end{aligned}
$$

The result below now follows by an easy induction on $n$.

COROLlaRY 3. Let $f:[a, b] \rightarrow \mathbf{R}$ be of bounded variation and $x_{i}:[a, b] \rightarrow \mathbf{R}$ ( $i=1, \ldots, n$ ) nonnegative, nondecreasing functions with continuous first derivative and $x_{i}(a)=0(i=1, \ldots, n)$.

If $0 \leq f(b) \leq f(x) \leq f(a)$ for all $x \in[a, b]$, then

$$
(f(a))^{n-1} \int_{a}^{b}\left(\prod_{i=1}^{n} x_{i}(t)\right)^{\prime} f(t) d t \geq \prod_{i=1}^{n} \int_{a}^{b} x_{i}^{\prime}(t) f(t) d t .
$$

If $0 \leq f(a) \leq f(x) \leq f(b)$ for all $x \in[a, b]$, the inequality is reversed. 
REMARK 5. Setting $a=0, b=1, g(x)=x^{a}, h(x)=x^{b}, f(0)=1$ in (4) gives Stolarsky's inequality, but with a weaker condition on $f$. In Theorem $\mathrm{C} f$ is monotonic, but in Theorem $4 f$ is of bounded variation and $f(x) \in[f(b), f(a)]$.

\section{Acknowledgement}

The authors are grateful to the referee for a number of helpful remarks.

\section{References}

[1] L. Maligranda, J. Pečarić and L. E. Persson, "Stolarsky's inequality with general weights", Proc. Amer. Math. Soc. 123 (1995) 2113-2118.

[2] D. S. Mitrinović and J. E. Pečarić, "Note on the Gauss-Winckler inequality", Anz. Österreich Akad. Wiss., Math.-Natur. Kl. 6 (1986) 89-92.

[3] D. S. Mitrinović, J. E. Pečarić and A. M. Fink, Classical and new inequalities in analysis (Kluwer, Dordrecht, 1993).

[4] J. Pečarić, “A reverse Stolarsky's inequality”, Amer. Math. Monthly 101 (1994) 565-567.

[5] J. Pečarić, "On Stolarsky's quotient”, Prilozi MANU (Skopje) 14 (2) (1993) 55-60.

[6] J. Pečarić and S. Varošanec, "Remarks on Gauss-Winckler's and Stolarsky's inequalities", Utilitas Math. 48 (1995) 233-241.

[7] K. B. Stolarsky, "From Wythoff's Nim to Chebyshev's inequality", Amer. Math. Monthly 98 (1991) 889-900.

[8] S. Varošanec and J. Pečarić, "Gauss' and related inequalities", Z. Anal. Anwendungen 14 (1994) 175-183.

[9] S. Varošanec and J. Pečarić, "Some integral inequalities with bounds for moments of distribution", J. Austral. Math. Soc., Ser. B 38 (1997) 325-335. 